EPJ Web of Conferences 60, 02003 (2013)

DOI: $10.1051 /$ epjconf/20136002003

(C) Owned by the authors, published by EDP Sciences, 2013

\title{
Tevatron Higgs results
}

\author{
Boris Tuchming ${ }^{1, \text { a }}$ for the CDF and D0 Collaborations. \\ ${ }^{1}$ CEA Saclay - Irfu/SPP - France.
}

\begin{abstract}
.
We present the combination of searches for the Standard Model Higgs boson, using up to $10 \mathrm{fb}^{-1}$ of $p \bar{p}$ collisions at $\sqrt{s}=1.96 \mathrm{TeV}$ collected with the CDF and D0 detectors at the Fermilab Tevatron collider. The major contributing channels are optimized for the main production modes, the associated production with a vector boson $(V H$, with $V=W, Z$ ), the vector boson fusion, and the gluon-gluon fusion, and the different decay modes $H \rightarrow b \bar{b}, H \rightarrow \tau^{+} \tau^{-}, H \rightarrow W^{+} W^{-}$, and $H \rightarrow \gamma \gamma$. A significant excess of events is observed in the mass range $115<M_{H}<140 \mathrm{GeV}$. The local significance corresponds to 3.0 standard deviations at $m_{H}=125 \mathrm{GeV}$, consistent with the mass of the new particle observed at the LHC. The observed signal strengths in all channels are consistent with the presence of a standard model Higgs boson of mass $125 \mathrm{GeV}$. We also present prospects for spin/parity tests to be performed in the $V H \rightarrow V b \bar{b}$ channels.
\end{abstract}

\section{Introduction}

Finding the standard model (SM) Higgs boson has been of the most topical goals of particle physics in the last decades.

Until 2000, direct searches were conducted at the CERN $e^{+} e^{-}$collider (LEP) that finally yielded the lower limit of $M_{H}>114.4 \mathrm{GeV}$ [1]. This exclusion limit and those reported hereafter are all defined at the 95\% C.L. After the end of searches at LEP, precision electroweak tests, including the $W$-boson mass mesurement from LEP, and the $W$-boson mass and top-quark mass measurements from the Tevatron Run I, contributed to constrain further the Higgs boson mass. In Summer 2002, the constraint from the electroweak fit read $M_{H}<193 \mathrm{GeV}$ [2]. This was greatly improved thanks to the top mass and $W$ mass measurements using the first data of Tevatron Run II, so that in Winter 2007, the indirect constraint was $M_{H}<$ $144 \mathrm{GeV}$ [3] which narrowed down substantially the expected mass range for the Higgs boson.

With the beginning of Run II of the Tevatron $p \bar{p}$ collider at $\sqrt{s}=1.96 \mathrm{TeV}$, a new cycle of searches started in 2002, that ended with the final Tevatron shutdown in September 2011. In 2008, the Tevatron Collaborations, $\mathrm{CDF}$ and D0, presented the first post-LEP-era direct constraint on the Higgs, excluding the mass $M_{H}=170 \mathrm{GeV}$ [4]. This constraint was extended over the years [5], and for example at Summer 2011 conferences the mass range from $156 \mathrm{GeV}$ to $177 \mathrm{GeV}$ was excluded [6].

With the Large Hadron Collider (LHC) a new era started. The 7 and $8 \mathrm{TeV} p p$ collision data from 20112012 allowed to explore a wide range of Higgs boson mass, and establish more stringent limits. Upper (lower)

a. e-mail: tuchming@cea.fr limits of 131 (122) $\mathrm{GeV}$ [7] and 128 (121.5) GeV [8] were obtained by the ATLAS and CMS Collaborations, respectively. But the perspective changed dramatically in 2012 with several announcements. On July 4th 2012, the ATLAS and CMS Collaborations reported excesses above background expectations at the five standard deviation (s.d.) level, consistent with the observation of a Higgs boson of $M_{H} \approx 125 \mathrm{GeV}[7,8]$. In the same week, the CDF and D0 Collaborations reported excesses above background expectations in the $H \rightarrow b \bar{b}$ search channels $[9,10]$. Combining CDF and D0 yields an excess at the three s.d. level, consistent with the production of a Higgs boson of mass $M_{H} \approx 125 \mathrm{GeV}$ [11]. With the discovery of the new particle, a measurement time started.

This proceedings discusses the final combined search results from the Tevatron collaborations, which represents the culmination of more than ten years of data analysis. For most of the channels, the full Run II dataset is used which corresponds to $\sim 10 \mathrm{fb}^{-1}$ of $p \bar{p}$ collisions per experiment after data quality requirements. The results are also interpreted to measure properties of the newly discovered particles: production rate in different modes and measurement of couplings to fermions and bosons. Most of these results have been recently submitted and accepted for publication (see Refs. [13-15] and references therein). The D0 internal combined results are discussed elesewhere in this proceedings [12].

\section{Higgs boson production and decay channels}

In the SM, the production cross-sections and the branching fractions as a function of the Higgs mass are well known. Over the mass range $90<M_{H}<200 \mathrm{GeV}$, 
the dominant production process is the gluon-gluon fusion $g g \rightarrow H(950 \mathrm{fb}$ at $125 \mathrm{GeV})$, followed by the associated production with a weak vector boson $p \bar{p} \rightarrow W H, p \bar{p} \rightarrow$ $\mathrm{ZH}$ (130 and $79 \mathrm{fb}$ at $125 \mathrm{GeV}$ ) and the weak vector boson fusion $p \bar{p} \rightarrow q q^{\prime} H(67 \mathrm{fb}$ at $125 \mathrm{GeV})$. The main decay modes for $M_{H}=125 \mathrm{GeV}$ are $H \rightarrow b \bar{b}(58 \%)$ and $H \rightarrow W^{+} W^{-}(22 \%)$. The most sensitive signatures are:

- one lepton $+\mathbb{E}_{T}+$ two $b$-jets $(W H \rightarrow \ell v b \bar{b})$

- no lepton $+\mathbb{E}_{T}+$ two $b$-jets $(Z H \rightarrow v \bar{v} b \bar{b})$

- two leptons + two $b$-jets $\left(Z H \rightarrow \ell^{+} \ell^{-} b \bar{b}\right)$

- two leptons $+\mathbb{E}_{T}\left(H \rightarrow W^{+} W^{-} \rightarrow \ell^{+} v \ell^{-} \bar{v}\right)$

Thus, the search for the SM Higgs at Tevatron mainly relies on $b$-tagging efficiency, good dijet mass resolution, high- $p_{T}$ lepton acceptance, good modeling of the $\mathbb{E}_{T}$, and good modeling of the $V+$ jet background. The Tevatron sensitivity to $V H \rightarrow V b \bar{b}$ is complementary to the LHC main discovery channels $(H \rightarrow \gamma \gamma, H \rightarrow Z Z$ ), which should help unravel the nature of the new particle.

The main sensitivity is given by the four channels presented above, but many other signatures are also considered to bring additional sensitivity and test the agreement with the SM expectations. For examples, Tevatron experiments have also looked for diphoton events $(H \rightarrow \gamma \gamma)$, associated production with top-quark pairs $(t \bar{t} H)$, lepton + $\mathbb{E}_{T}+$ dijet signature (from $H \rightarrow W W$ ), trilepton signatures (e.g. from $W H \rightarrow W W W$ ), same charge dilepton signatures (e.g. from $W H \rightarrow W W W$ ), quadrilepton signatures (e.g. from $\mathrm{ZH} \rightarrow \ell^{+} \ell^{-} W W \rightarrow \ell^{+} \ell^{-} \ell^{+} \ell^{-} v \bar{v}$ ), and tau-based signatures (e.g. from $W H \rightarrow \ell v \tau^{+} \tau^{-}$or $\left.W H \rightarrow q \bar{q} \tau^{+} \tau^{-}\right)$.

\section{Analysis strategy}

Over the course of Run II, both collaborations have followed the same strategy to optimize the analyses and improve their sensitivity faster than expected from just accumulating more and more data.

- Acceptance is maximized by lowering kinematic requirements on leptons, by including different lepton reconstruction categories, by accepting events from all possible triggers, and by optimizing $b$-jet tagging with more and more sophisticated multivariate techniques (MVA).

- MVA techniques are widely used in all channels as they provide typically $25 \%$ more sensitivity than just using single kinematic discriminant such as the dijet mass for the $V H \rightarrow V b \bar{b}$ channels. The improved sensitivity obtained thanks to MVA can be assessed by eye in Fig. 1, which compares the most discriminant variables (dijet mass) and the MVA in the D0 $\mathbb{E}_{T}+b \bar{b}$ search channel [16].

Each MVA combines into a single discriminant many variables, which include for example variables describing the event topology, the lepton and jet kinematics, the quality of leptons, and the relation between leptons/jets and $\mathbb{E}_{T}$. All channels at Tevatron employ at least one MVA, optimized for each different Higgs boson mass hypothesis. For most of the analyses several MVAs
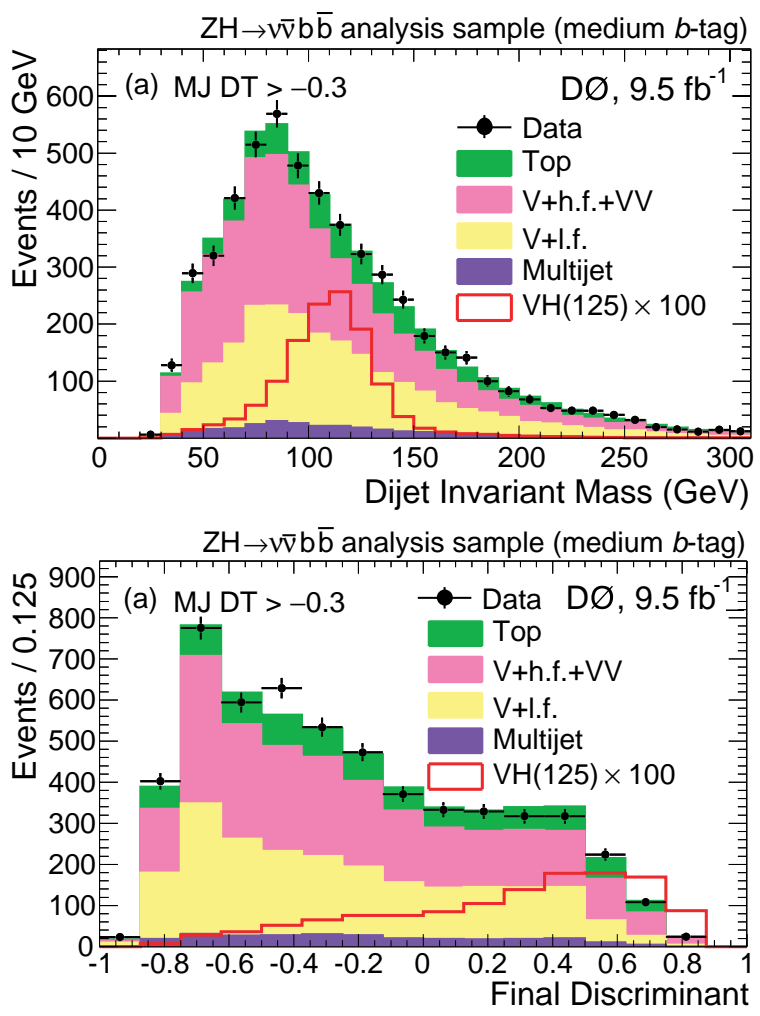

Figure 1. Top, distribution of the dijet invariant mass of candidate events in the D0 $\mathbb{E}_{T}+b \bar{b}$ search channel. Bottom, distributions of the final MVA at the same selection stages.

trained specifically against different backgrounds bring additional sensitivity.

The MVA techniques are also employed for object identification ( $b$-jets, leptons, photons) and for energy correction to $b$-jets. For example the usage of the CDF HOBIT [18] b-tagging algorithm in the final published $V H \rightarrow V b \bar{b}$ analyses provides an enhancement of $20 \%$ in $b$-tagging efficiency per jet.

- Another way of achieving better sensitivity to signal, consists in splitting the search channels into subchannels according to jet multiplicity, $b$-tagging content, lepton flavor or lepton quality. Dedicated MVA are also trained to split analyses into subchannels enhanced or enriched in specific backgrounds. Using subchannels with different signal-over-background ratio $(s / b)$ maximizes discriminating power, allows sensitivity to different signal production modes, and give more handles and lever-arm to control backgrounds and systematic uncertainties. As an example, Fig. 2 shows the final MVA output for the three $b$-tagging categories of the CDF $\mathbb{E}_{T}+b \bar{b}$ analysis [17].

- The data are employed as much as possible to control backgrounds and validate search methods. Instrumental backgrounds, such as jets faking leptons, photons faking electrons, charge mismeasurements, and tail of $\mathbb{E}_{T}$ resolution are measured in dedicated control samples. Background enriched samples are also employed to check modeling of specific background processes. Fi- 

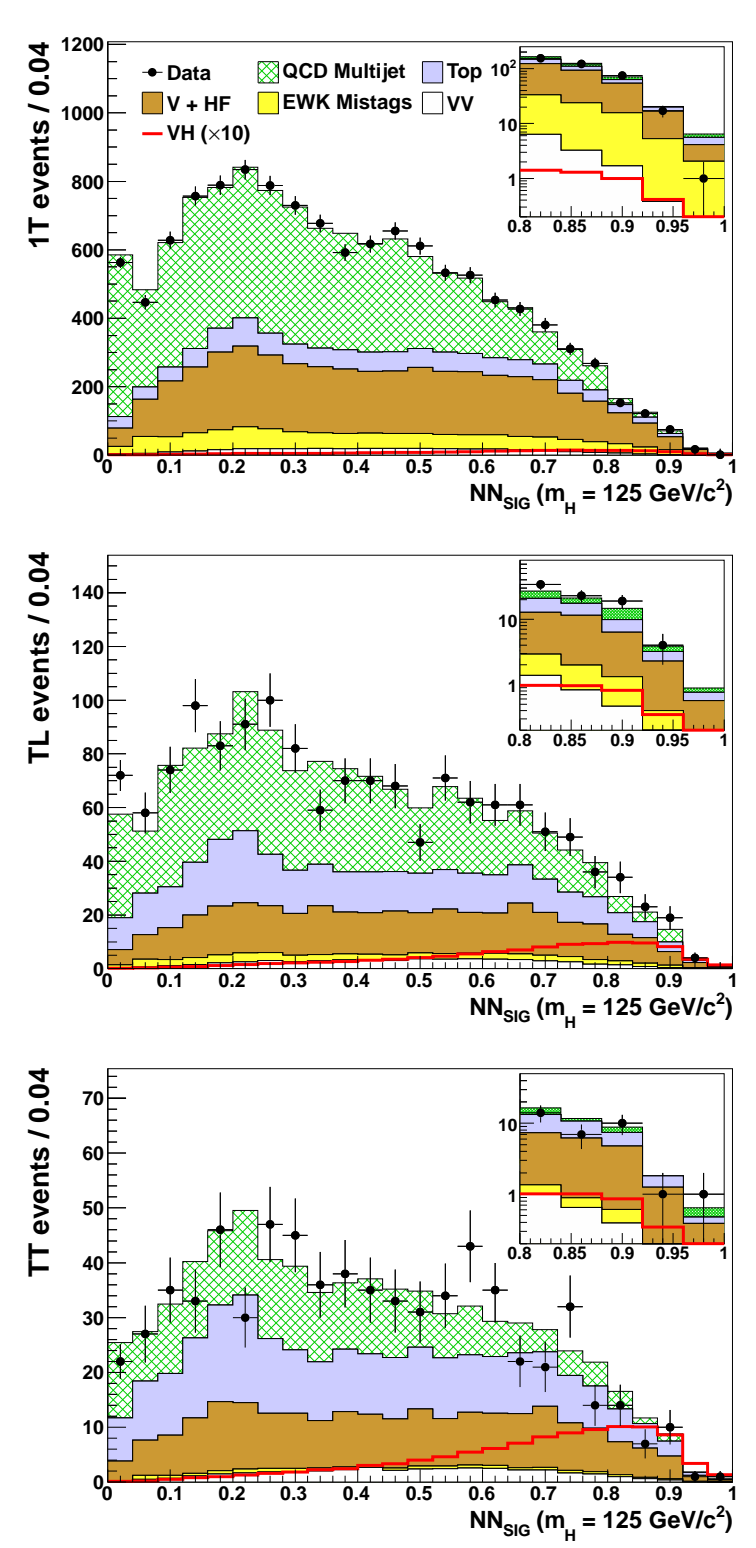

Figure 2. Final MVA discriminant in $\mathrm{CDF} \mathbb{E}_{T}+b \bar{b}$ analysis for the three subchannels defined according to the $b$-tagging of jets: top, only one jet is tightly tagged (1T); middle, one jet is tightly tagged, and the other loosely tagged (TL); and bottom, both jets are tightly tagged (TT).

nally, the same analysis techniques, namely the same kind of MVA, the same subchannels and the same treatment of systematic uncertainties are employed to measure production rates of known standard model candles such $p \bar{p} \rightarrow W^{+} W^{-} \rightarrow \ell^{+} v \ell^{-} \bar{v}$, or $V Z \rightarrow V b \bar{b}$. At D0, the measured cross section $\sigma\left(W^{+} W^{-}\right)=11.6 \pm 0.7 \mathrm{pb}^{-1}$ is in agreement with the NNLO prediction of $11.3 \pm$ $0.7 \mathrm{pb}^{-1}$. The combined CDF+D0 measured cross section $\sigma(W W+W Z)=3.0 \pm 0.6$ stat \pm 0.7 syst $\mathrm{pb}^{-1}$ is in agreement with $\mathrm{SM}$ prediction of $4.4 \pm 0.3 \mathrm{pb}^{-1}$. Figure 3 shows the background subtracted dijet mass distribution in highest $b$-tagging categories used in this later measurement.

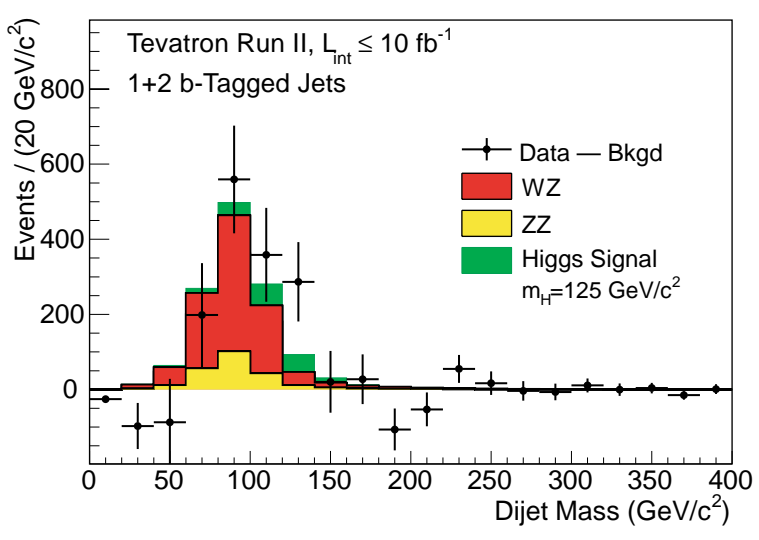

Figure 3. Background subtracted dijet invariant mass distribution of the $V Z$ analyses in the high $b$-tag purity categories.

\section{Result of the search}

The combination of all search channels for $M_{H}=$ $125 \mathrm{GeV}$ can be visualized in Fig. 4, where the background subtracted distribution of the final discriminant for all channels are sorted as a function of $s / b$ and then added. An excess of events in the highest $s / b$ bins is observed. Figure 5 shows the log-likelihood ratio (LLR) testing the signal-plus-background over the background-only hypothesis and computed for different test masses.

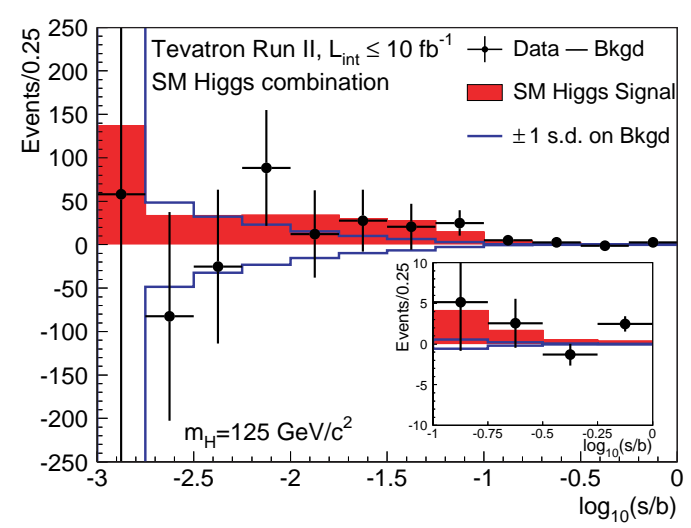

Figure 4. Background subtracted distribution of events using the final discriminant of all channels sorted in bins of $s / b$, for $M_{H}=125 \mathrm{GeV}$.

A significant signal-like excess in the mass range between 115 and $140 \mathrm{GeV}$ is observed. The background p-value of that excess corresponds to $3.0 \mathrm{~s}$.d. for $M_{H}=$ $125 \mathrm{GeV}$, as shown in Fig. 6. That excess arises from both CDF (2.0 s.d.) and D0 (1.7 s.d) data, as expected from the presence of a standard model Higgs boson of mass $125 \mathrm{GeV}$.

The results are interpreted in terms of limits on the Higgs boson production measured in units of the expected SM production. They are shown in Fig. 7 as a function of the Higgs boson mass. The combined CDF and D0 results have almost reached the exclusion sensitivity for the full range $[90,185] \mathrm{GeV}$. More precisely the ex- 


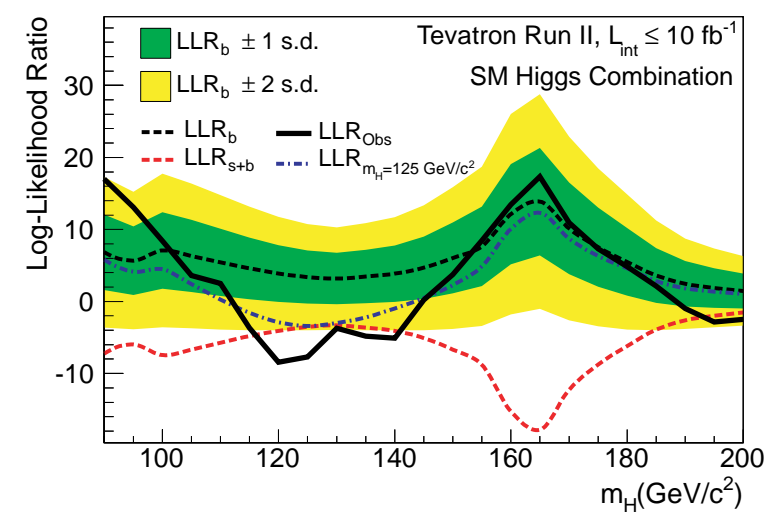

Figure 5. LLR computed as a function of Higgs boson mass hypothesis.

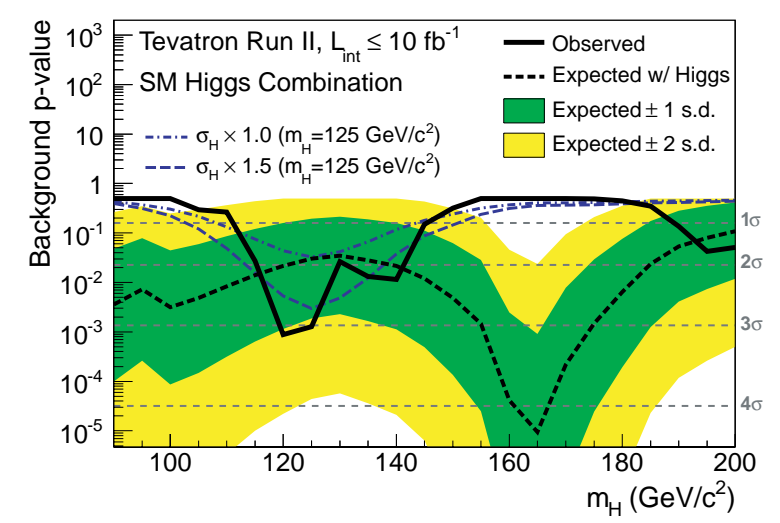

Figure 6. Background p-value as a function of the Higgs boson mass hypothesis.

pected excluded regions at $95 \%$ C.L. are $[90,120] \mathrm{GeV}$ and $[140,184] \mathrm{GeV}$. Because of the excess observed in the low mass region, the actual observed exclusion ranges are smaller: [90,109] $\mathrm{GeV}$ and $[149,182] \mathrm{GeV}$. For $M_{h}=125 \mathrm{GeV}$, the observed (expected) production limits are $2.44 \times \mathrm{SM}(1.06 \times \mathrm{SM})$.

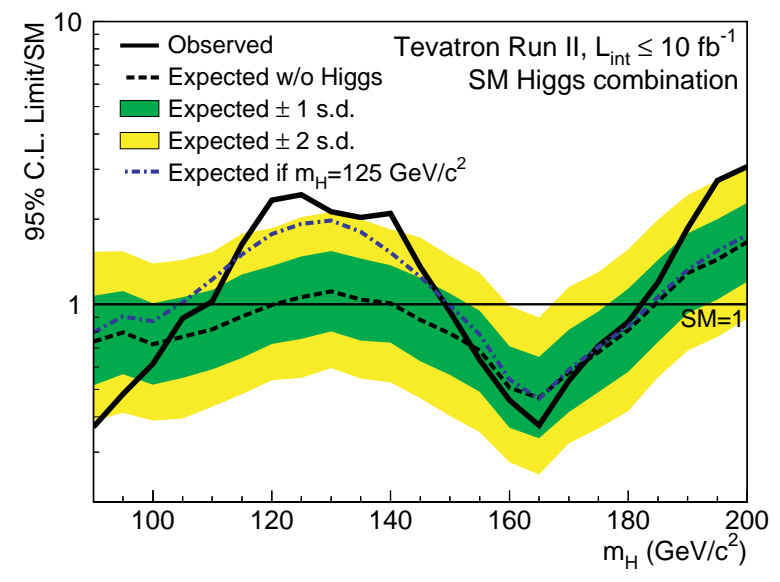

Figure 7. Limits on the SM Higgs boson production as a function of the mass hypothesis.

\section{Searches beyond the standard model}

The different SM channels can be used to search for physics beyond the SM.

The Tevatron Collaborations interpret their results in fermiophobic framework in which the Higgs couplings to fermions are heavily suppressed, thus suppressing the gluon-gluon fusion process. In this search the main modes are the $H \rightarrow \gamma \gamma$ and $H \rightarrow W W$ search channels, as both branching ratios are enhanced. The combined observed (expected) exclusion resulting from these channels is $M_{H}<116 \mathrm{GeV}\left(M_{H}<135 \mathrm{GeV}\right)$.

Another interpretation is performed within the context of a fourth generation of fermions. In this framework the existence of heavy colored quarks enhances the gluongluon fusion by approximately a factor of nine, thus the search is performed in the $g g \rightarrow H \rightarrow W W$ channels only. The absence of significant excess of data events allows to exclude the mass range of $121<M_{H}<225 \mathrm{GeV}$, while the expected exclusion range is $118<M_{H}<270 \mathrm{GeV}$, as shown in Fig. 8.

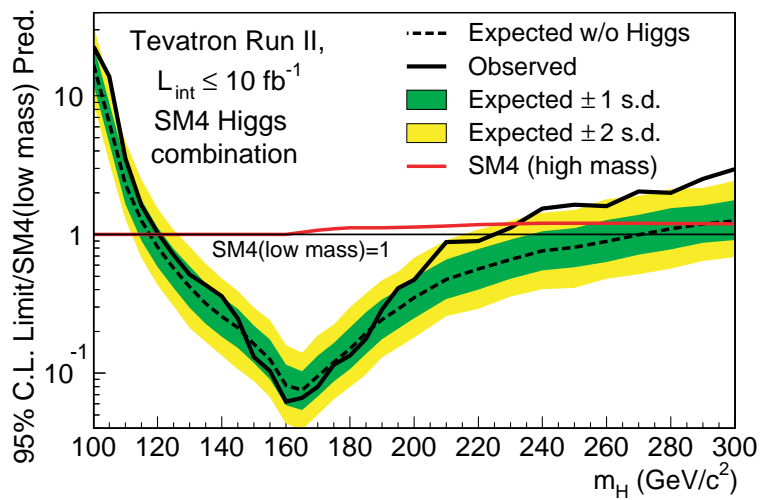

Figure 8. Limits on the Higgs boson production in the context of a fourth generation of fermions as a function of the Higgs boson mass.

\section{Higgs boson studies}

\subsection{Measurement of production rates}

The SM search channels can be separately combined to measure the yield in the different modes: $H \rightarrow b \bar{b}, H \rightarrow$ $\tau^{+} \tau^{-}, H \rightarrow W^{+} W^{-}$, and $H \rightarrow \gamma \gamma$. The best fits to the data are summarized in Table 1 and displayed in Fig. 9. The overall production rate of $1.44_{-0.56}^{+0.59} \times \mathrm{SM}$ is obtained, compatible with the SM Higgs boson of mass $125 \mathrm{GeV}$. The modes with sizable signal-like excesses relative to the background-only hypothesis are $V H \rightarrow V b \bar{b}$ and $H \rightarrow$ $W^{+} W^{-}$, as expected from the SM Higgs boson.

\subsection{Measurement of couplings to fermions and bosons}

We assume a SM-like Higgs particle of $125 \mathrm{GeV}$, with no additional particle in loops and no invisible decays. The SM couplings to fermions and vector bosons 
are scaled by three numbers, $\kappa_{f}, \kappa_{W}$, and $\kappa_{Z}$. For example the $W H \rightarrow W b \bar{b}$ yield is then scaled by $\frac{\kappa_{f}^{2} \kappa_{W}^{2}}{\kappa_{H}^{2}}$, where the terms in the numerator correspond the scaling of the incoming and outgoing partial width, while the denominator is a global scaling factors for the total Higgs boson width.

A fit to the data is performed by separating and scaling properly the contributions from the different production and decay modes. Note that in this procedure, only a few modes exhibit some dependence on the relative sign between the coupling scale factors, due to interferences between diagrams. The most important effect arises from the interference between $W$ loops and top-quark loops for the $H \rightarrow \gamma \gamma$ partial width: $\Gamma(H \rightarrow \gamma \gamma)=\Gamma(H \rightarrow$ $\gamma \gamma)_{S M} \times\left(1.28 \kappa_{W}-0.28 \kappa_{f}\right)^{2}$.

The results of the fit are shown in Fig. 10: when $\kappa_{f}$ is let floating (with flat prior) the best fit region is around $\left(\kappa_{W}, \kappa_{Z}\right)=(1.25, \pm 0.90)$; assuming custodial symmetry

\begin{tabular}{lcc|c} 
& $\mathrm{CDF}[13]$ & $\mathrm{D} 0[14]$ & $\mathrm{CDF}+\mathrm{D} 0[15]$ \\
\hline \hline$R_{\mathrm{fit}}(\mathrm{SM})$ & $1.54_{-0.73}^{+0.77}$ & $1.40_{-0.88}^{+0.92}$ & $1.44_{-0.56}^{+0.59}$ \\
\hline$R_{\mathrm{fit}}\left(H \rightarrow W^{+} W^{-}\right)$ & $0.00_{-0.00}^{+1.78}$ & $1.90_{-1.52}^{+1.63}$ & $0.94_{-0.83}^{+0.85}$ \\
$R_{\mathrm{fit}}(V H \rightarrow V b \bar{b})$ & $1.72_{-0.87}^{+0.92}$ & $1.23_{-1.17}^{+1.24}$ & $1.59_{-0.72}^{+0.69}$ \\
$R_{\mathrm{fit}}(H \rightarrow \gamma \gamma)$ & $7.81_{-4.42}^{+4.61}$ & $4.20_{-4.20}^{+4.60}$ & $5.97_{-3.12}^{+3.39}$ \\
$R_{\mathrm{fit}}\left(H \rightarrow \tau^{+} \tau^{-}\right)$ & $0.00_{-0.00}^{+8.44}$ & $3.96_{-3.38}^{+4.11}$ & $1.68_{-1.68}^{+2.28}$ \\
$R_{\mathrm{fit}}(t \bar{t} H \rightarrow t \bar{t} b \bar{b})$ & $9.49_{-6.28}^{+6.60}$ & - & - \\
\hline \hline
\end{tabular}

Table 1. Best fit to the data of the Higgs boson production (in unit of the SM Higgs boson production), assuming $M_{H}=125 \mathrm{GeV}$, for the different channels and their combination.

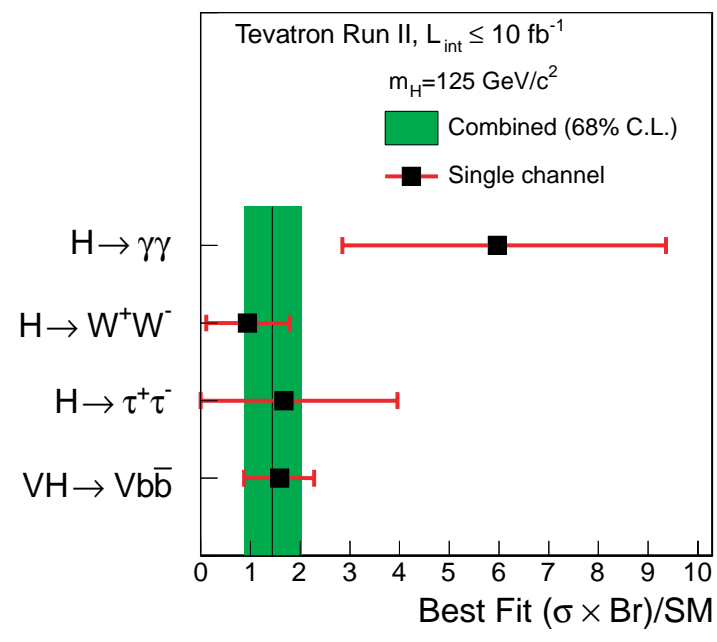

Figure 9. Best fit to the data of the Higgs boson production in different channels, assuming $M_{H}=125 \mathrm{GeV}$.
$\left(\kappa_{W}=\kappa_{Z}=\kappa_{V}\right)$, the two preferred regions are around $\left(\kappa_{V}, \kappa_{f}\right)=(1.05,-2.40)$ and $\left(\kappa_{V}, \kappa_{f}\right)=(1.05,2.30)$.
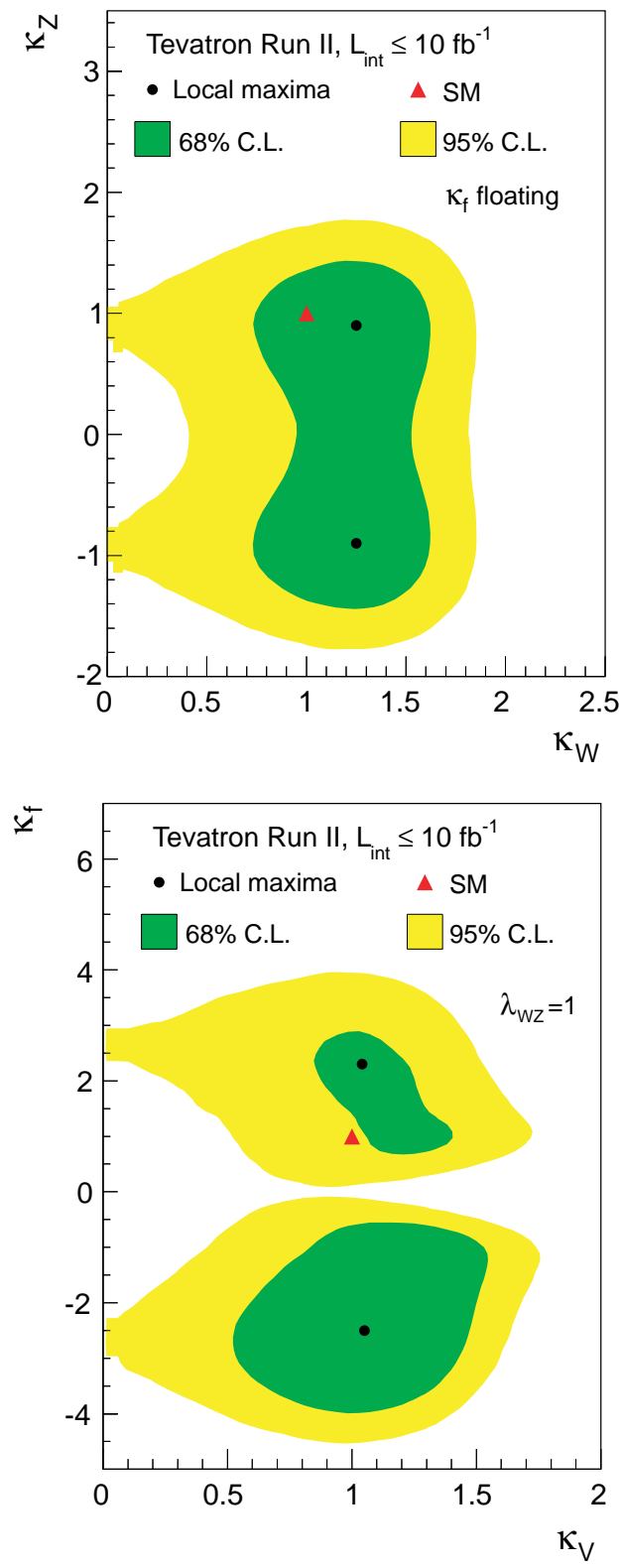

Figure 10. Posterior density probability for the coupling scale factors, $\kappa_{f}, \kappa_{W}$, and $\kappa_{Z}$, obtained under different assumptions.

These 2-dimensions results can be turned into one dimension constraints: assuming $\kappa_{W}=\kappa_{f}=1$, the best-fit value is $\kappa_{Z}= \pm 1.05_{-0.55}^{+0.45}$; assuming $\kappa_{Z}=\kappa_{f}=1$, the best-fit $68 \%$ confidence intervals are defined by $\kappa_{W}=-1.27_{-0.29}^{+0.46}$ and $1.04<\kappa_{W}<1.51$; assuming $\kappa_{W}=\kappa_{Z}=1$, the best-fit value is $\kappa_{f}=-2.64_{-1.30}^{+1.59}$; and by letting $k_{f}$ floating with a flat prior, the custodial symmetry is tested and the best fit value for the ratio $\lambda_{W Z}=\frac{\kappa_{W}}{\kappa_{Z}}$ reads $\lambda_{W Z}=1.24_{-0.42}^{+2.34}$. All these results are in agreement with the SM expectations within their uncertainties. 


\subsection{Spin and parity tests}

In general spin/parity of a particle affects angular distributions of its decay products, but also cross-section behavior near production threshold. This later property can be exploited at Tevatron in the $V H \rightarrow V b \bar{b}$ search modes. The spectra of the effective center-of mass energy, $\sqrt{\hat{s}}$, of $V H \rightarrow V b \bar{b}$ events are expected to be quite different under different spin and parity hypothesis $\left(0^{-}, 0^{+}\right.$, or $\left.2^{+}\right)$for $H$ [19]. This can be exploited by using as main discriminant observable the overall mass (or transverse mass for final state with neutrinos) of the candidate events. The D0 analysis is discussed elsewhere in this proceedings [20]. No measurement has been performed yet, but each Tevatron Collaboration is expected to release results for the forthcoming conferences.

\section{Conclusion}

After ten years of excellent performance for the Tevatron collider and the CDF and D0 experiments, both Tevatron collaborations combine their final results on the SM Higgs boson searches. They almost achieve exclusion sensitivity over the full range [90 - 185] GeV, and exclude at 95\% C.L. the range of mass $90<M_{H}<109 \mathrm{GeV}$ and $149<M_{H}<182 \mathrm{GeV}$. Interpretation of results beyond standard model yields the limits $M_{H}<116 \mathrm{GeV}$ for a fermiophobic Higgs, and $121<M_{H}<225 \mathrm{GeV}$ in the context of a fourth generation of fermions.

In the search for the SM Higgs boson, both CDF and D0 observe an excess of signal-like events in the low mass range $115<M_{H}<140 \mathrm{GeV}$, compatible with the experimental resolution. Its combined significance is 3.0 s.d. for $M_{H}=125 \mathrm{GeV}$ and it arises mainly from the $H \rightarrow b \bar{b}$ and $H \rightarrow W^{+} W^{-}$channels, as expected from the SM Higgs boson. The measured production rate of $1.44_{-0.56}^{+0.59} \times \mathrm{SM}$ and the measured couplings are compatible with a Higgs boson of $125 \mathrm{GeV}$. The experiments have also good prospects to probe the spin/parity of the Higgs-like particle of $125 \mathrm{GeV}$ in the $V H \rightarrow V b \bar{b}$ modes.

\section{Acknowledgments}

We thank the Fermilab staff and technical staffs of the participating institutions for their vital contributions. We acknowledge support from the DOE and NSF (USA), ARC (Australia), CNPq, FAPERJ, FAPESP and FUNDUNESP (Brazil), NSERC (Canada), NSC, CAS and CNSF (China), Colciencias (Colombia), MSMT and GACR (Czech Republic), the Academy of Finland, CEA and CNRS/IN2P3 (France), BMBF and DFG (Germany), DAE and DST (India), SFI (Ireland), INFN (Italy), MEXT (Japan), the Korean World Class University Program and NRF (Korea), CONACyT (Mexico), FOM (Netherlands), MON, NRC KI and RFBR (Russia), the Slovak R\&D Agency, the Ministerio de Ciencia e Innovación, and Programa Consolider-Ingenio 2010 (Spain), The Swedish Research Council (Sweden), SNSF (Switzerland), STFC and the Royal Society (United Kingdom), the A.P. Sloan Foundation (USA), and the EU community Marie Curie Fellowship contract 302103 .

\section{References}

[1] R. Barate et al., Phys. Lett. B 565, 61 (2003).

[2] [Electroweak Working Group and SLD Heavy Flavor Group Collaborations], (2002) hep-ex/0212036.

[3] E. Barberis,"Top mass measurement at the Tevatron," O. Stelzer-Chilton, "First Run II Measurement of the $W$ Boson Mass with CDF," in Proceedings of the 42nd Rencontres De Moriond On Electroweak Interactions And Unified Theories, edited by J. M. Frere, L. Iconomidou-Fayard, F. Montanet, J. Tran Thanh Van. Hanoi, Gioi Publ., (2007).

[4] The CDF and D0 Collaborations and the Tevatron New Physics and Higgs Working Group, (2008) arXiv:0808.0534.

[5] T. Aaltonen et al. [CDF and D0 Collaborations], Phys. Rev. Lett. 104, 061802 (2010).

[6] The CDF and D0 Collaborations and the Tevatron New Physics and Higgs Working Group, arXiv:1107.5518 (2012).

[7] G. Aad et al. [ATLAS Collaboration], Phys. Lett. B 716, 1 (2012).

[8] S. Chatrchyan et al. [CMS Collaboration], Phys. Lett. B 716, 30 (2012).

[9] T. Aaltonen et al. [CDF Collaboration], Phys. Rev. Lett. 109, 111802 (2012).

[10] V. M. Abazov et al. [D0 Collaboration], Phys. Rev. Lett. 109, 121802 (2012).

[11] T. Aaltonen et al. [CDF and D0 Collaborations], Phys. Rev. Lett. 109, 071804 (2012).

[12] E. Chapon, "Combined search for the SM Higgs Boson at D0", this proceedings

[13] T. Aaltonen et al. [CDF Collaboration], arXiv:1301.6668 (2013), accepted by Phys. Rev. D.

[14] V. M. Abazov et al. [D0 Collaboration], arXiv:1303.0823 (2013), accepted by Phys. Rev. D.

[15] T. Aaltonen et al. [CDF and D0 Collaborations], arXiv:1303.6346, accepted by Phys. Rev. D.

[16] V. M. Abazov et al. (D0 Collaboration), Phys. Lett. B 716, 285 (2012).

[17] T. Aaltonen et al. (CDF Collaboration), arXiv:1301.4440 (2013), accepted by Phys. Rev. D.

[18] J. Freeman et al., Nucl. Instrum. Meth. A 697, 64 (2013)

[19] J. Ellis, D. S. Hwang, V. Sanz and T. You, JHEP 1211, 134 (2012)

[20] B. Tuchming, "Spin/parity of Higgs-like particle at D0", this proceedings. 